\title{
Limitations of Transient Power Loads on DEMO and Analysis of Mitigation Techniques
}

\author{
Francesco Maviglia $^{\mathrm{ab}^{*}}$, Gianfranco Federici ${ }^{\mathrm{a}}$, Gerhard Strohmayer ${ }^{\mathrm{c}}$, Ronald Wenninger ${ }^{\mathrm{ac}}$, Christian \\ Bachmann ${ }^{\mathrm{a}}$, Raffaele Albanese ${ }^{\mathrm{b}}$, Roberto Ambrosino ${ }^{\mathrm{d}}$, Muyuan $\mathrm{Li}^{\mathrm{c}}$, Vincenzo P. Loschiavo ${ }^{\mathrm{b}}$, \\ Jeong-Ha You ${ }^{\mathrm{c}}$, Louis Zani ${ }^{\mathrm{e}}$.
}

${ }^{a}$ EUROfusion Consortium, PPPT Department, Garching, Boltzmannstr. 2, Germany

${ }^{b}$ Consorzio CREATE, Univ. Napoli Federico II - DIETI, 80125 Napoli, Italy

${ }^{c}$ Max-Planck-Institut fur Plasmaphysik, Garching, Boltzmannstr. 2, Germany

${ }^{d}$ Consorzio CREATE Univ. Napoli Parthenope, Naples, Italy

${ }^{e}$ CEA, F-13108 St Paul-Lez-Durance, France

*Corresponding author: francesco.maviglia@euro-fusion.org

\begin{abstract}
In this paper is presented the assessment of the operational space for the present DEMO standard water cooled tungsten mono-block divertor targets and copper alloy heat sink, under static and transient heat loads. A transient thermo-hydraulic analysis has been performed using the code RACLETTE, which allowed a broad parametric scan of the target geometry and coolant conditions. The limiting factors considered were the coolant Critical Heat Flux (CHF), and the materials temperatures. The second part of the work is devoted to the study of the plasma strike point sweeping as a mitigation technique for the divertor power exhaust. The RACLETTE code was used to evaluate the impact of a large set of sweeping frequencies and amplitudes. A reduced subset of cases, which complied with the desired results, were benchmarked with a 3D FEM model, leading to a reduction of the heat flux to the coolant, up to a factor $\sim 4$, and a lower materials temperature, for an incident heat flux in the range [15-30] $\mathrm{MW} / \mathrm{m}^{2}$. Finally a preliminary assessment was performed on the installed power required for the sweeping, the AC losses in the superconductors and the thermal fatigue analysis. No evident show stoppers were found.
\end{abstract}

Keywords: DEMO, tokamak, divertor, power exhaust, mono-block, divertor target.

\section{Introduction}

The operating conditions of the DEMO (DEMOnstration fusion power reactor) divertor will be more demanding than the one presently expected in ITER due to more severe material and technical limitations, as the higher neutron fluence and longer pulses. ITER divertor [1-2], on which technology is based the present DEMO design, is made of tungsten mono-block divertor targets and copper alloy heat sink cooling pipe, with water used as coolant with an inlet temperature of $100^{\circ} \mathrm{C}$. It is designed to work with steady state heat flux loads up to $10 \mathrm{MW} / \mathrm{m}^{2}$ during plasma burning operations, and to withstand slow transient heat loads up to $20 \mathrm{MW} / \mathrm{m}^{2}$ for $\sim 10 \mathrm{~s}$, including a safety margin equal to 1.7 , with actual pipe burnout limit equal to $\sim 34 \mathrm{MW} / \mathrm{m}^{2}$. The DEMO standard single null configuration, presently considered, based on the described ITER technology, will have reduced margins in the heat flux removal capability, due to the higher coolant inlet operational temperature, required to avoid the copper alloys embrittlement at high neutron irradiation [3-4]. Although a new investigation on copper alloys irradiation is in programed for the next years in DEMO relevant conditions, the present knowledge suggests to operate the water coolant at a temperature $\geq$ $200^{\circ} \mathrm{C}$, which decrease the heat removal performances and decrease the operational space margins for the expected power input range.
The present estimated heat loads due to the charged particles in DEMO, in absence of any radiation, are in the range of $150 \mathrm{MW} / \mathrm{m}^{2}$, which is way outside the present technological limits. Techniques to reduce the heat load to the divertor, essential also for ITER, are currently been explored in the main fusion devices, including the control of the plasma into regimes where the major part of the power to the divertor is radiated [5], and the divertor detachment [6]. Failure of the above controls may lead to sudden increase of heat flux with potentially destructive events for the divertor, and for this reason they need to be studied to evaluate the adequate counteraction, in terms of control strategies and reaction time.

In the first part of the paper is presented a transient thermo-hydraulic analysis, performed using the code RACLETTE [7-8], which allowed a broad parametric scan of the target geometry and coolant conditions, using as input a wide range of incident heat flux. The second part of the work is devoted to the study of the plasma strike point sweeping as a mitigation technique for the divertor power exhaust. A parametric scan was performed on a set of sweeping frequencies and amplitudes at different levels of incident power heat flux. A narrowed subset of cases was further validated using a 3D FEM code. Finally a preliminary assessment was carried out on the installed power needed for the sweeping cases considered, the perturbations on the plasma shape, the $\mathrm{AC}$ losses induced in the closest 
superconductive coils, and the thermal-fatigue. No evident show stoppers were found.

\section{Thermo-hydraulic analysis}

A transient thermo-hydraulic analysis has been performed to evaluate the heat removal capabilities of the divertor targets due to steady state and under slow transient heat loads under the expected DEMO conditions. The analysis was performed using the code RACLETTE [7-8], a relatively simple and versatile 1D model, with 2D corrections, which includes in good details most of the surface heat transfer processes such as evaporation, melting, and radiation, and their interaction with the PFC block thermal response and the coolant behavior. The use of RACLETTE allowed to quickly run a broad parametric scan of the target geometry and coolant conditions, such as the tungsten (W) armor thickness, the copper alloy pipe and interlayer diameter and length, the mono-block pitch between two pipes, and the coolant pressure, velocity and inlet temperature. The input power flux footprint used in the model is according to the latest prediction for DEMO [9]. One of the limiting factor considered was the coolant $\mathrm{CHF}$, which is the maximum heat flux that can be removed before the coolant begins to boil, determining the pipe burnout, leading to the destructive event of water in the machine. The other limiting factors are the mono-block materials temperature operational range under the expected DEMO neutron fluence.

\section{Simulation of transient loads handling}

The transient analysis was performed giving a step function as input power, simulating a sudden increase of the incident heat flux, from a value below the one that would lead to the reaching of the CHF, i.e. between zero and the expected steady stave value $10 \mathrm{MW} / \mathrm{m}^{2}$, to a value that exceed the $\mathrm{CHF}$, for example to mimic a loss of control in the divertor detachment. The output calculated consisted in the time taken for the divertor to reach the $\mathrm{CHF}$ or the material melting, in function of the final value of the input step function. Two scans will be presented in this work.

\subsection{Scan of the coolant inlet temperature from ITER to present predicted DEMO ranges}

The first case presented considers the difference on heat removal capability in function of the water coolant inlet temperature, in a range from ITER $\left(100^{\circ} \mathrm{C}\right)$ to the present DEMO predictions $\left(\geq 200^{\circ} \mathrm{C}\right)$. The latter limit is due to the current knowledge, based on [3-4], and is present to avoid the copper alloys embrittlement at high neutron irradiation at temperatures lower than the value indicated. Investigation on irradiated materials will be performed in the next years under relevant DEMO conditions. The analysis was performed considering the standard model of divertor mono-block currently considered, i.e. with a $5 \mathrm{~mm}$ tungsten armor and $28 \mathrm{~mm}$ distance between the center of two adjacent blocks, a copper alloy ( $\mathrm{CuCrZr}$ ) pipe (acting as heat sink) thickness of $1.5 \mathrm{~mm}$ and diameter of $12 \mathrm{~mm}$, and a coolant pressure of $4 \mathrm{MPa}$, a velocity $12 \mathrm{~m} / \mathrm{s}$ and a turbulence promotion factor 1.9 (swirl tube). The initial input value used in the input function before the power density step was considered to be zero, in the analysis presented in Fig1. The heat removal capability is reduced by increasing the inlet water coolant temperature from $100^{\circ} \mathrm{C}$ (ITER like), where $\mathrm{CHF}$ is reached for input power values $>50 \mathrm{MW} / \mathrm{m}^{2}$, identified by the solid vertical lines, to a temperature of $200^{\circ} \mathrm{C}$ (DEMO like), where the CHF is reached for input power values of $24 \mathrm{MW} / \mathrm{m}^{2}$. In the first case, the ITER-like condition, the melting of the tungsten surface, represented by dashed lines, is reached for an input power of $43 \mathrm{MW} / \mathrm{m}^{2}$, i.e. before the $\mathrm{CHF}$, while for DEMO the CHF is reached much before the melting. The outcome of this analysis is that:

- Melting is not the driving criterion for DEMO, as it is for ITER, for slow transients as the one presented.

- CHF is in DEMO the limiting factor.

- CHF margin is substantially reduced in DEMO.

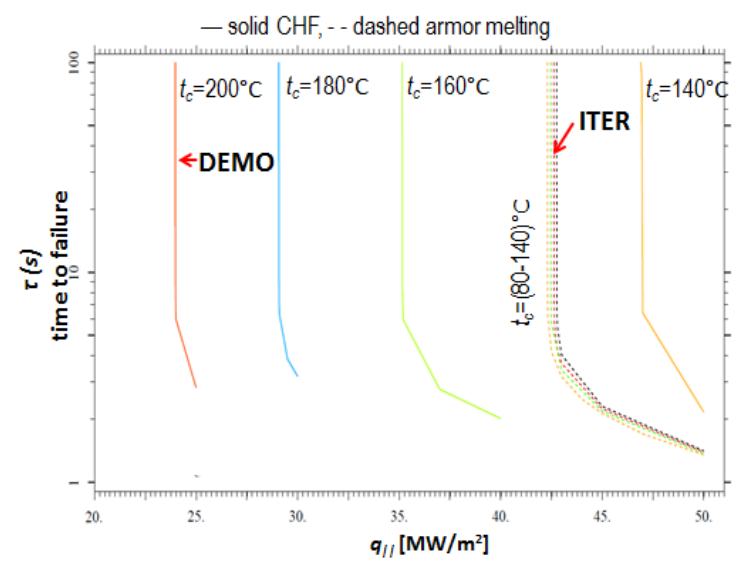

Fig.1. Time to failure $\tau(s)$ is shown in a logarithmic scale on a $\mathrm{y}$-axis, due to step function from 0 to the value of $\mathrm{MW} / \mathrm{m}^{2}$ shown on the $\mathrm{x}$-axis, for coolant temperature in the range $100^{\circ} \mathrm{C}$ (ITER-like) to $200^{\circ} \mathrm{C}$ (DEMO-like). The solid lines represent the reaching of the $\mathrm{CHF}$, the dotted the reaching of melting.

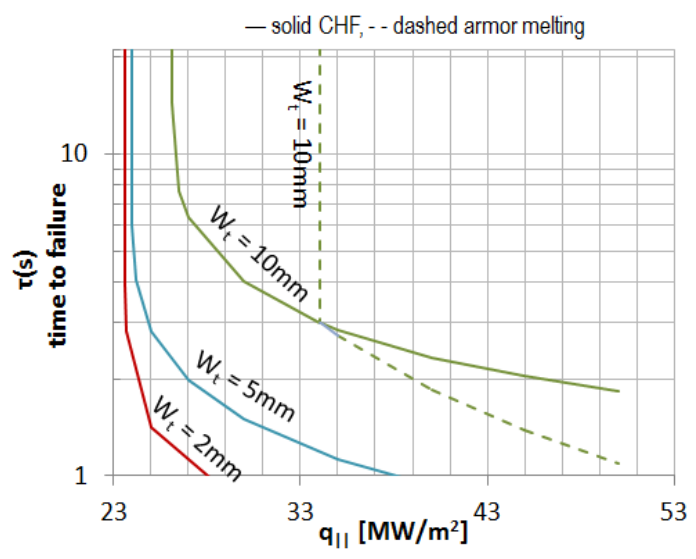

Fig.2. Time to failure for coolant temperature of $200^{\circ} \mathrm{C}$ (DEMO-like), for tungsten armour thickness in the range $2 \mathrm{~mm}$ to $10 \mathrm{~mm}$. All other parameters are as in Fig.1.

\subsection{Scan of the tungsten armor thickness}

The second case presented considers the value of the water coolant temperature fixed at $200^{\circ} \mathrm{C}$, and the other parameters as in the previously described case, except 
the armor thickness, which is varied in the range $(2,5$, $10) \mathrm{mm}$. This is done to evaluate the tradeoff between being limited by the $\mathrm{CHF}$ or the tungsten melting temperature in DEMO-like conditions. The Fig.2 shows the results using an armor thickness of $(2,5,10) \mathrm{mm}$, which correspond to an increase in the CHF to a value of $(23,24,26) \mathrm{MW} / \mathrm{m}^{2}$. This is a minor improvement in the maximum CHF value, but it is interesting to notice the time needed to reach this value. For instance in the plot in correspondence of the $y$ axis value of $3 \mathrm{~s}$ to the failure, the $10 \mathrm{~mm}$ thick tungsten armor can withstand a jump of incident power up to $34 \mathrm{MW} / \mathrm{m}^{2}$, while at the $2 \mathrm{~s}$ value this become up to $45 \mathrm{MW} / \mathrm{m}^{2}$, with contemporary staring melting starting from $34 \mathrm{MW} / \mathrm{m}^{2}$. This may allow a time margin of $3 \mathrm{~s}$, in the first case, to detect and react to an increasing of the allowable steady state incoming power, or $2 \mathrm{~s}$, in the second case, with the sacrifice of the melting of some tungsten surface. This time may be sufficient for instance to bring back the heat flux down to a tolerable lever or shutting down the plasma. This would avoid the destructive event of the cooling pipe burnout, with consequent water in the machine, which may be a potentially fatal event for DEMO. Thicker tungsten armor may also compensate the material erosion during the divertor lifetime operation, while it would have as drawback a higher operating surface temperature.

\section{Divertor heat loads mitigation techniques}

The eventuality of a sudden increase of the influx power to the divertor represents a serious treat for both ITER and the presently expected DEMO burning plasma phase. This may be caused by physics reasons, or technical failure of a control system which may, for instance, leads to a loss of detachment regime. As it is shown in the previous section, this may bring to a destruction of the targets due to reaching of the CHF limit, in a time scale of the order from a fraction of second to few seconds, depending of the maximum heat flux reached and the target design. In the present experimental tokamak are already present emergency strategies to protect the machine if certain limits are hit, which allow, for instance, executing a different scenario or, eventually, to start a shutdown procedure which quickly bring to the plasma current termination. An example of such system for the JET tokamak is reported in [10-11]. In DEMO the task to detect an increase of incoming heat power may be more demanding due to the limited type of diagnostic capability which can be installed close enough to the plasma in a highly neutron radiated environment. In the present DEMO design, a pulsed machine with two hours burning phase, it will be highly desirable to be able to demonstrate continuous operations with as less interruptions as possible due to unforeseen events, to guarantee a reliable electricity production. This would avoid also the need to start up and shut down the plasma, being both these phases demanding in term the amount of electricity required from the grid. In this respect, in long pulsed machines, it would be highly desirable to be able to react to temporary increase of heat flux to the divertor, above the sustainable limits, with maneuvers that mitigate the higher power loads, avoiding the destruction of the targets, while the control systems try to recover, if possible, the desired plasma regime, or ultimately to safely shut down the machine. In the next paragraphs will be presented the analysis carried out on the strike point sweeping technique.

\subsection{Strike point sweeping operational space}

The plasma strike point sweeping is a mitigation technique which is used in the present devices to reduce the thermal loads on the divertor targets both in tokamaks [12] and stellarators [13]. It consists in a periodic movement of the strike points, which are the contact point of the plasma separatrix flux-lines with the divertor, with predefined amplitude and frequency. This periodic movement reduces the localized thermal load by spreading the total energy over a larger area. A sensitivity analysis was performed using the code RACLETTE on a broad range of parameters, hereafter reported:

\section{Input parameter for the outer strike point:}

- Incident heat flux $\mathrm{q}_{\|}$peak range $(15,30) \mathrm{MW} / \mathrm{m}^{2}$, according the DEMO latest predicted footprint [9]

\section{Sweeping parameters:}

- Amplitude range (peak to peak) $(5,10,20,40) \mathrm{cm}$

- Frequency $(0.1,0.2,0.5,1.0,2.0,5.0) \mathrm{Hz}$.

Mono-block model geometric parameter:

- Tungsten armor thickness $(5,10) \mathrm{mm}$.

- Other parameters are as presented in paragraph 3.1.

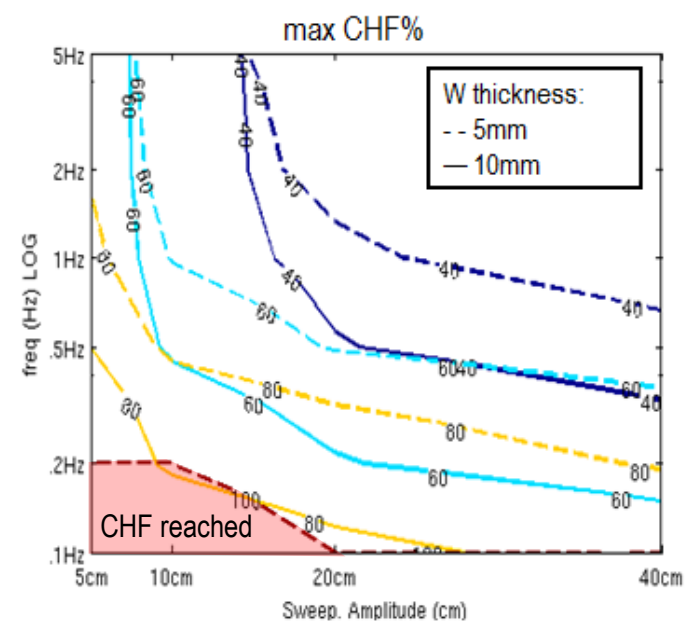

Fig.3. Contour plot of the CHF percentage reached in function of the sweeping frequency and amplitude, respectively on the $y$ and $x$ axis, for armor thickness of $5 \mathrm{~mm}$ and $10 \mathrm{~mm}$ and input heat flux of $30 \mathrm{MW} / \mathrm{m}^{2}$.

Given the non-symmetric nature of the incident heat flux curve [9] it was find out, in a preliminary 2D analysis, that the highest temperature is reached close to the corner right position of the sweeping range for the outer strike point. Although the exact position of the temperature peak is a function of the frequency and amplitude, it was decided to consider the position where the maximum temperature was calculated for a case in 
the middle of the frequency and amplitude operational space considered.

The outputs considered in the analysis were:

- the percentage to the $\mathrm{CHF}$, as ratio between heat flux to the coolant over the $\mathrm{CHF}$ value, where $100 \%$ means that the CHF limit is reached, with cooling pipe burn out;

- the tungsten armor surface temperature, with its melting limit at $3422^{\circ} \mathrm{C}$, and a recrystallization temperature around $1200^{\circ} \mathrm{C}$, above which a significant reduction of the strength of tungsten was observed in the heat flux tests of divertors [14], and major cracks appears on its surface;

- the heat sink $\mathrm{CuCrZr}$ pipe maximum temperature, with a limit at $350^{\circ} \mathrm{C}$, above which the material start softening/aging [15].

The results presented are relative to the most severe case analyzed, with input power of $30 \mathrm{MW} / \mathrm{m}^{2}$, as to simulate, for instance, a partial loss of control of plasma detachment. Fig. 3 shows the results of the parametric scan, where in the $x$ and $y$ axes are reported respectively the sweeping amplitude and frequency, while the contour line are the maximum percentage to the $\mathrm{CHF}$ reached. If a safety factor of 1.7 to the $\mathrm{CHF}$ (CHF represented by the $100 \%$ contour line) is considered, the resulting $60 \%$ to the CHF operational space is achieved either for frequency equal to $0.5 \mathrm{~Hz}$ and amplitudes greater than $>20 \mathrm{~cm}$, or for amplitudes equal to $10 \mathrm{~cm}$, and frequencies greater than $1 \mathrm{~Hz}$, for armor thickness of $5 \mathrm{~mm}$, which represents the worst case. With similar sweeping parameters also the maximum temperature of the $\mathrm{CuCrZr}$ heat sink stays below $350^{\circ} \mathrm{C}$, and no melting is reached on the tungsten surface in the sweeping operational space considered, although the recrystallization limit of $1200^{\circ} \mathrm{C}$ is satisfied only for the sweeping frequencies greater than $2 \mathrm{~Hz}$ and amplitudes greater that $30 \mathrm{~cm}$, for the most demanding case, which is this time the one with armor thickness of $10 \mathrm{~mm}$. The union of these results, together with consideration on other technical limitations, which will be discussed later, as the installed power needed to perform the sweeping, the AC losses on the closes superconductors, helped to determines operational space of interest and to narrow down the sweeping parameters.

\subsection{D FEM model of divertor mono-block}

A 3D FEM model was developed to analyze a subset of cases of interest, coming from the broader scan presented in the previous paragraph. The details of the model used in this analysis are described in [16]. The analysis was limited to a mono-block with armor thickness of $5 \mathrm{~mm}$, input heat flux power of $(15,30)$ $\mathrm{MW} / \mathrm{m} 2$, and seeping parameters of $(0.5,1) \mathrm{Hz}$, and amplitudes of $(5,20) \mathrm{cm}$ peak to peak. The results obtained were in good agreement with the RACLETTE code, within $15 \%$.

The Fig.4a shows the comparison of the tungsten surface temperature in the 4 sweeping cases with the stationary case, for the $15 \mathrm{MW} / \mathrm{m}^{2}$ input heat-flux. No tungsten melting is reached in any of the cases considered. The maximum tungsten surface temperature decrease from $\sim 1290^{\circ} \mathrm{C}$, in the stationary case, down to $\sim 550^{\circ} \mathrm{C}$, in the sweeping case at a frequency of $1 \mathrm{~Hz}$ and amplitude of $20 \mathrm{~cm}$, below the recrystallization temperature of $\sim 1200^{\circ} \mathrm{C}$. Fig. $4 \mathrm{~b}$ shows the same simulation but compares the maximum $\mathrm{CuCrZr}$ heat sink temperature which decreases from $400^{\circ} \mathrm{C}$, in the stationary case, above the copper softening/aging limit, down to $270^{\circ} \mathrm{C}$, in the $1 \mathrm{~Hz} / 20 \mathrm{~cm}$ sweeping, within the desired limits. The maximum heat flux to the coolant also decrease considerably from $25 \mathrm{MW} / \mathrm{m}^{2}$, larger than the incident heat flux because of the peaking factor due to the circular shape of the pipe, down to $6 \mathrm{MW} / \mathrm{m}^{2}$, with a reduction of a factor 4 .

a)

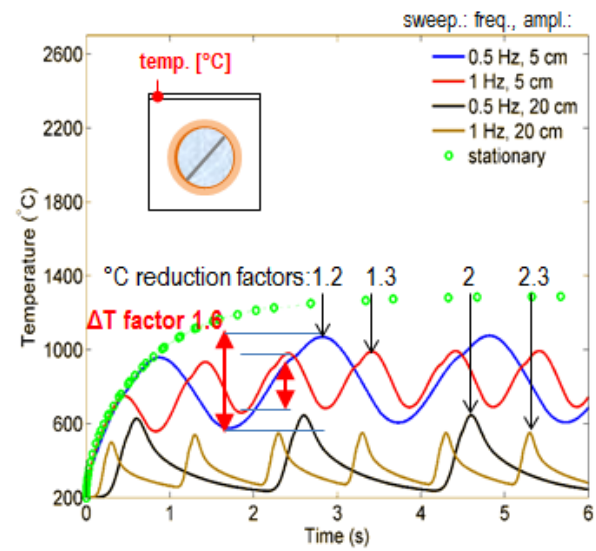

b)

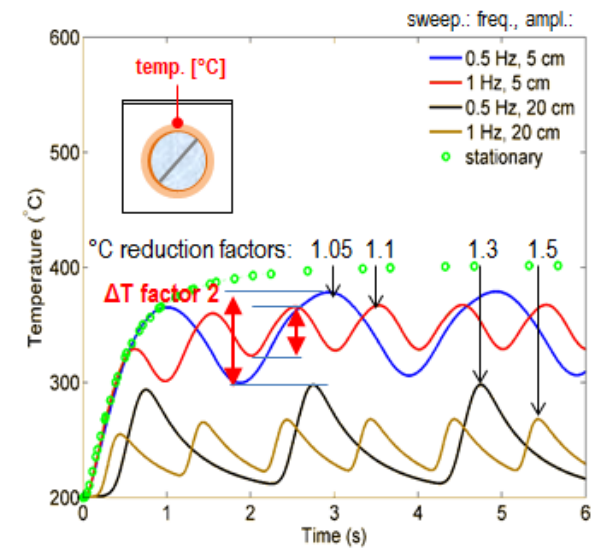

Fig.4. Maximum temperature of a) tungsten armour, and b) copper alloy heat sink, for the stationary and sweeping cases.

\subsection{Thermal fatigue analysis}

In this paragraph are presented the results on the analysis performed on the impact of sweeping parameters on the accumulated equivalent plastic strain in the copper interlayer, the details of which can be found in [16], while in previous papers was reported that nearly no cyclic plastic deformation is accumulated in the cooling tube [17]. The results show that the fatigue lifetime increases as the peak flux densities increase. Furthermore if the sweeping frequency is increased of a factor 2 , from $0.5 \mathrm{~Hz}$ to $1 \mathrm{~Hz}$, the fatigue lifetime is increased at least by a factor of 4, extending the operating time for interlayer. The estimated operating time for the sweeping cases considered is reported in the table 1 . 


\begin{tabular}{|l||l|l|}
\hline Sweeping ampl./freq. & $0.5 \mathrm{~Hz}$ & \multicolumn{1}{|c|}{$1 \mathrm{~Hz}$} \\
\hline \hline $5 \mathrm{~cm}$ & $66 \mathrm{~h}$ & $236 \mathrm{~h}$ \\
\hline $20 \mathrm{~cm}$ & $42 \mathrm{~h}$ & $134 \mathrm{~h}$ \\
\hline stationary (1 pulse of $2 \mathrm{~h})$ & $12553 \times 2$ \\
\hline
\end{tabular}

Table.1. Predicted fatigue lifetime (hours) considering the total loading time for the sweeping cases considered.

By applying the sweeping technique with the parameters studied in this work, the result is an operating time up to $\sim 236$ hours. This result makes the sweeping suitable as an emergency maneuver to rescue the targets from a destructive event, in case an increase heat-flux is detected. It is instead not suitable as a standard operation during the whole plasma burning phase, presently designed to be 2 hours for each DEMO pulse, in the present sweeping and amplitude operational range considered. Another consideration is that sweeping the high heat flux load in a larger target area reduces the loading time for each individual mono-block element, reducing the localized thermal-induced material degradation, e.g. recrystallization, the possible creep effect and tungsten sputtering.

\section{Sweeping power requirements and $\mathrm{AC}$ losses}

A preliminary analysis was performed on the installed power required to perform the strike point sweeping, by using the CREATE L [18] model of a standard DEMO equilibrium, with an aspect ratio of 3.1, plasma current of 20MA, $95 \%$ elongation of 1.5 , major radius of $8.7 \mathrm{~m}$ and toroidal field at the magnetic axis of 5.8T. Two sweeping copper coils, connected in antiseries, were preliminary considered located $80 \mathrm{~cm}$ behind the divertor. The limitation due to the nuclear damages was not considered in this work, but the position chosen could in principle allow the possibility to provide a sufficient shielding and allow the maintenance. An alternative solution could be represented by the use of saddle coils in each toroidal sector, integrated in the divertor cassette, and replaceable with the time scale of the DEMO divertor. A sinusoidal current with amplitude of $240 \mathrm{kA}$ (170 kA rms) and a frequency from 0 to $5 \mathrm{~Hz}$ was considered. The figure of $240 \mathrm{kA}$ the nominal current for the internal VS coils in ITER. While the required power scales quadratically, most other relevant parameters, such as strike point motion, plasma motion, magnetic field, etc., have a linear dependence on the sweeping coil current. The power needed for sweeping in the above conditions with the current considered is:

- active power of $0.30 \mathrm{MW}$ at $0.2 \mathrm{~Hz}, 3.3 \mathrm{MW}$ at $1 \mathrm{~Hz}$;

- reactive power of $3.5 \mathrm{MVAr}$ at $0.2 \mathrm{~Hz}, 16 \mathrm{MW}$ at $1 \mathrm{~Hz}$.

At the very low frequency considered this would provide a sweep of up to $26 \mathrm{~cm}$ peak-to-peak on the outer strike-point and $19 \mathrm{~cm}$ on the inner one.

Estimation on the AC losses in the superconductive coils closer to the divertor was also performed. Those are defined as the eddy currents and hysteresis phenomena due to the varying the magnetic field, which heat the superconductive coils decreasing their cooling capabilities. At low frequency, up to $1 \mathrm{~Hz}$, the performance is at least $19 \mathrm{~cm}$ sweep peak-to-peak for both strike points, yielding a maximum $\mathrm{dB} / \mathrm{dt}$ of about $15 \mathrm{mT} / \mathrm{s} \mathrm{rms}$ at $0.2 \mathrm{~Hz}$ and $60 \mathrm{mT} / \mathrm{s} \mathrm{rms}$ at $1 \mathrm{~Hz}$.

The maximum temperature increase is of $0.03 \mathrm{~K}$, for $\mathrm{F}=1 \mathrm{~Hz}$. This did not include the hysteresis losses that might also play a role. If a likely total of $0.1 \mathrm{~K}$ is considered, this is comparable with the $\mathrm{AC}$ losses due to the DEMO scenario, which are of the order of $0.3 \mathrm{~K}$, then they are not negligible. The first macro analysis on ITER PF6 with present DEMO sweeping parameters do not excludes the possibility of using sweeping method at the expense of acceptable decrease of $T$ margin in the closest superconductive coil, but a definitive assessment will be carried out in a dedicated thermo-hydraulic study.

\section{Conclusions}

\section{Acknowledgments}

This work has been carried out within the framework of the EUROfusion Consortium and has received funding from the Euratom research and training programme 2014-2018 under grant agreement No 633053. The views and opinions expressed herein do not necessarily reflect those of the European Commission."

\section{References}

[1] M. Merola, et al., ITER plasma-facing components, Fusion Engineering and Design 85 (2010) 2312-2322.

[2] A. Raffray, et al., High heat flux components - readiness to proceed from near term fusion systems to power plants, Fusion Engineering and Design 85 (2010) 93-108.

[3] S.A. Fabritsiev, et al., Journal of Nuclear Materials (1996)

[4] S.A. Fabritsiev, et al., Plasma Devices and Operat., (1997)

[5] Kallenbach A. et al 2012 Nucl. Fusion 52122003

[6] Wischmeier M. et al 2011 Proc. 19th Int. Conf. on PlasmaSurface Interactions in Controlled Fusion, J. Nucl. Mater. 415 S523-9

[7] A. Raffray, G. Federici, Journal of Nucl. Materials (1997).

[8] G. Federici, A. Raffray, RACLETTE: a model for evaluating the thermal response of plasma facing components to slow high power plasma transients. Part I: Theory and description of model capabilities, Journal of Nuclear Materials Vol. 244, I 2, 1 April 1997, 85-100.

[9] T. Eich, et al, 2013 Nucl. Fusion 53093031.

[10] G. De Tommasi, et al., "Plasma position and current control system enhancements for the JET ITER-like wall", Fusion Engineering and Design 89 (2014) 233242.

[11] D.F. Valcárcel, et al., "The JET real-time plasma-wall load monitoring system”, Fusion Engineering and Design 89 (2014) 243-258.

[12] G. Ambrosino, et al., "Plasma strike-point sweeping on JET tokamak with the extreme shape controller", Plasma Science, IEEE Transactions on 36 (2008) 834-840.

[13] R. Koenig, et al., The divertor program in stellarators, Plasma Physics and Controlled Fusion 44 (2002) 2365.

[14] G. Pintsuk, et al., Qualification and post-mortem 
characterization of tungsten mock-ups exposed to cyclic high heat flux loading, Fusion Engineering and Design 88 (2013) $1858-1861$.

[15] M. Miskiewicz, J.-H. You et al.,"Impact of plastic softening of over-aged $\mathrm{CuCrZr}$ alloy heat sink tube on the structural reliability of a plasma-facing component", Fus Eng Des Vol 83, I 1, Jan 2008, Pages 66-71.

[16] M. Li, et al., "Thermal response of a water-cooled divertor target under sweeping heat flux loads", to be submitted.

[17] M. Li, E. Werner, J.-H. You, Low cycle fatigue behavior of iter-like divertor target under demo-relevant operation conditions, Fus. Eng. and Des. 90 (2015) 88-96.

[18] R. Albanese and F. Villone, "The linearized CREATE-L plasma response model for the control of current, position and shape in tokamaks," Nucl.Fusion, vol. 38, no. 5, pp. 723-738, 1998. 